\title{
Hypothesis
}

\section{Intravascular radiotherapy: restenosis and more?}

At the end of the 1970s balloon angioplasty emerged as a new hope in the treatment of coronary atherosclerosis. ${ }^{1} \mathrm{~A}$ decade later, the implantation of endovascular stents ${ }^{2}$ did not only reduce the number of acute complications of percutaneous coronary interventions, but also the incidence of restenosis. ${ }^{3}$ Now, as we begin the new millennium, intravascular radiotherapy promises to lower the restenosis rate further and has been shown to be especially effective in treatment of in-stent restenosis. ${ }^{4}$

Restenosis develops as a response to injury, mediated by a complex interaction of an inflammatory process, thrombus formation, proliferation and migration of smooth muscle cells (SMCs), expression of growth factors, matrix synthesis, and finally vascular remodelling. ${ }^{5}$ The antiproliferative effects of ionising radiation, which are widely used in treating various benign and malignant proliferative diseases, are based on the interaction of primary ( $\beta$ radiation) or secondary ( $\gamma$ radiation) electrons with biochemical bonds in cellular DNA, ${ }^{6}$ and on subsequent changes in gene expression that finally lead to a reduction of cell growth. In cell culture and various animal models numerous radioactive devices (wires, seeds, stents, liquid filled balloons) have been proven to reduce SMC proliferation successfully. ${ }^{7}$ In some studies even a decreased degree of constrictive remodelling was noted, which was attributed to a reduction of the adventitial reaction to balloon injury (myofibroblast proliferation). ${ }^{8}$ Most of the previously conducted clinical trials on vascular brachytherapy successfully lowered restenosis rates with both $\beta$ and $\gamma$ radiation $^{49}$; however, aneurysm development after high dose irradiation, ${ }^{10}$ and the "candy wrapper" stenosis observed after implantation of radioactive stents, remain important limitations that deserve careful examination in both experimental and clinical studies.

Restenosis is clinically rather benign and mostly presents as stable angina, whereas the final, often fatal event in atherosclerosis is plaque rupture in haemodynamically non-significant lesions. ${ }^{11}$ The unstable plaque consists of SMCs, macrophages, and foam cells ("lipid core"), typically calcified and necrotic features, and a thin fibrous cap which is prone to fissuring. Macrophages play a major role in unstable angina by various means. They synthesise matrix degrading metalloproteinase ${ }^{12}$ which destabilise the fibrous cap and thus facilitate plaque rupture induced by shear stress. Macrophages also release proinflammatory cytokines, which in part stimulate SMC proliferation, and tissue factor ${ }^{11}$, the initiating protein in the extrinsic coagulation cascade. At plaque rupture, the exposure of circulating blood to thrombogenic proteins in the subendothelium rapidly induces thrombosis. The site of plaque rupture, most frequently the plaque shoulder, shows evidence of an ongoing inflammatory process, marked by $\mathrm{T}$ lymphocytes, macrophages, and focally raised temperature. ${ }^{13}$ The clinical impact of inflammation in the outcome of acute coronary syndromes was recently elucidated by reports on the predicting value of $\mathrm{C}$ reactive protein in patients with unstable angina and acute myocardial infarction. ${ }^{15}$

We hypothesise that local irradiation may be used to calm the inflammatory process in the unstable atherosclerotic plaque. Although dose finding, risk stratification, and assessment of the underlying biological mechanisms are still subject to intense investigations, radiotherapy for treatment of inflammation has been integrated into clinical routine for decades. ${ }^{16}$ Being an empirically based form of antiphlogistic therapy, it is used in a variety of benign diseases, such as hydradenitis, parotitis, panaritium, epicondylitis, or heterotopic ossification after orthopaedic surgery. ${ }^{16-18}$ Recently it has been shown that radiotherapy modulates the nitric oxide pathway in stimulated macrophages, ${ }^{19}$ which may in part explain the mitigated inflammatory reaction after irradiation of diseases involving macrophages, such as heterotopic ossification. ${ }^{20}$ Regarding vascular brachytherapy, Rubin and colleagues ${ }^{21}$ observed a pronounced reduction of invading monocytes and adventitial macrophages after irradiation of balloon injured rat carotids, corresponding to decreased neointima formation. This report emphasises the anti-inflammatory potential of vascular brachytherapy, although the mechanisms of macrophage reduction are not yet clear. In unstable plaques, reduction of macrophages might firstly result in a lower amount of metalloproteinases. Cessation of their matrix degrading activity will in consequence lessen the degree of the fibrous cap's destabilisation. Secondly, the emerging decrease in tissue factor synthesis will additionally moderate thrombogenicity after eventual plaque fissuring, and thirdly, the persisting macrophages will be impaired in certain pathways of inflammatory reactions, leading to a general mitigation of the inflammatory process in the arterial wall. Taken together, macrophage reduction may suppress the occurrence of plaque rupture and thrombosis, which mainly determine the patient's risk for development of acute coronary syndromes.

Thorough testing in experimental settings will be necessary to clarify the feasibility of using vascular brachytherapy in acute coronary interventions, and to determine the possible negative sequelae of macrophage reduction (for example, promoted formation of the thrombogenic lipid core by apoptotic macrophages, ${ }^{22}$ upregulated expression of matrix degrading and/or thrombogenic factors by apoptotic cells, and induction of inflammatory processes in non-atherosclerotic vessel segments $\left.{ }^{23}\right)$. Although there has been no evidence of an increased apoptosis rate after experimental vascular brachytherapy so far, ${ }^{24}$ these considerations have to be taken into account regarding differences between stable and unstable, or primary and restenotic, lesions.

If irradiation modulates inflammatory processes in the unstable plaque, it could lead to a significant decrease in major adverse cardiac events. This may open a new indication for intravascular brachytherapy as a novel local anti-inflammatory treatment for unstable angina.

PAUL WEXBERG

MICHAEL GOTTSAUNER-WOLF

Department of Cardiology, Clinic for Internal Medicine II,

University of Vienna, Währinger Gürtel 18-20, A-1090 Vienna Austria email:pwexberg@pop3.kard.akh-wien.ac.at

1 Gruentzig A, Senning A, Siegenthaler W. Nonoperative dilatation of coronary artery stenosis: Percutaneous transluminal coronary angioplasty. $N \mathrm{Engl}$ ary artery stenosis: Per

2 Sigwart U, Puel J, Mirkovitch V, et al. Intravascular stents to prevent occlusion and restenosis after transluminal angioplasty. $N$ Engl f Med 1987;316: $13-17$. 
3 Fischman D, Leon M, Baim D, et al. A randomized comparison of coronary-stent placement and balloon angioplasty in the treatment of corSe. N Engl f Med 1994;331:496-501.

4 Teirstein P, Massullo V, Jani S, et al. Catheter-based radiotherapy to inhibit restenosis after coronary stenting. N Engl F Med 1997;336:1697-703.

5 Gottsauner-Wolf M, Moliterno D, Lincoff A, et al. Restenosis - an open file. Clin Cardiol 1996;19:347-56.

6 Hall E. Radiobiology for the radiologist. Philadelphia: JB Lippincott, 1994.

7 Bertrand O, Mongrain R, Lehnert S, et al. Intravascular radiation therapy in atherosclerotic disease: promises and premises. Eur Heart $\mathcal{F}$ 1997;18:138595.

8 Wilcox J, Waksman R, King S, et al. The role of the adventitia in the arterial response to angioplasty: The effect of intravascular radiation. Int $\mathcal{F}$ Radiat Oncol Biol Phys 1996;36:789-96.

9 King S, Williams D, Chougule P, et al. Endovascular $\beta$-radiation to reduce restenosis after coronary balloon angioplasty. Results of the Beta Energy Restenosis Trial (BERT). Circulation 1998;97:2025-30.

10 Condado J, Waksman R, Gurdiel O, et al. Long-term angiographic and clinical outcome after percutaneous transluminal coronary angioplasty and

11 Fuster V, Fallon J, Badimon J, et al. The unstable atherosclerotic plaque: clinical significance and therapeutic intervention. Thromb Haemost 1997; 78:247-55.

12 Brown D, Hibbs M, Kearney $M$, et al. Identification of $92-\mathrm{kD}$ gelatinase in human coronary atherosclerotic lesions. Association of active enzyme synthesis with unstable angina. Circulation 1995;91:2125-31.

13 van der Wal A, Becker A, van der Loow C, et al. Site of intimal rupture or erosion of thrombosed coronary atherosclerotic plaques is characterized by an inflammatory process irrespective of the dominant plaque morphology. Circulation 1994;89:36-44.

14 Casscells W, Hathorn B, David M, et al. Thermal detection of cellular infiltrates in living atherosclerotic plaques: possible implications for plaque rupture and thrombosis. Lancet 1996;347:1447-51.
15 Morrow D, Rifai N, Antman E, et al. C-reactive protein is a potent predictor of mortality independently of and in combination with troponin $\mathrm{T}$ in acute coronary syndromes: a TIMI 11A substudy. Thrombolysis in myocardial infarction. F Am Coll Cardiol 1998;31:1460-5.

16 Trott K. Therapeutic effects of low radiation doses. Strahlenther Onkol 1994; 170:1-12.

17 Heyd R, Schopohl B, Kirchner J, et al. Strahlentherapie der Epiconylopathia humeri. Dtsch Med Wochenschr 1997;122:247-52.

18 Kienapfel H, Koller M, Wüst A, et al. Prevention of heterotopic bone formation after total hiparthroplasty: a prospective randomised study comparing postoperative radiotherapy with indomethacin medication. Arch Orthop Traum Surg 1999;119:296-302.

19 Hildebrandt G, Seed M, Freemantle C, et al. Mechanisms of the anti-inflammatory activity of low-dose radiation therapy. Int $\mathcal{F}$ Radiat Biol 1998;74:367-78.

20 Moraes J, Moraes F. Effect of a persistent inflammatory process on experimental heterotopic ossification. The influence of macrophages. Braz $\mathcal{F} M e d$ Biol Res 1993;26:53-66.

21 Rubin P, Williams J, Riggs P, et al. Cellular and molecular mechanisms of radiation inhibition of restenosis. Part I: role of the macrophage and platelet-derived growth factor. Int f Radiat Oncol Biol Phys 1998;40:92941

22 Libby $\mathrm{P}$, Geng Y, Aikawa M, et al. Macrophages and atherosclerotic plaque stability. Curr Opin Lipidol 1996;7:330-5.

23 Kallfass E, Kramling HJ, Schultz Hector S. Early inflammatory reaction of the rabbit coeliac artery wall after combined intraoperative (IORT) and external (ERT) irradiation. Radiother Oncol 1996;39:167-78

24 Waksman R, Rodriguez J, Robinson K, et al. Effect of intravascular irradiation on cell proliferation, apoptosis, and vascular remodeling after balloon overstretch injury of porcine coronary arteries. Circulation 1997;96:194452.

\section{IMAGES IN CARDIOLOGY}

\section{Fatal unruptured myocardial abscesses}

A 67 year old man was admitted with a two week history of intermittent lower chest pain. He had been treated for a bacteriologically confirmed Escherichia coli urinary tract infection six months previously. On admission he was apyrexial, normotensive, and an echocardiogram showed a $1-2 \mathrm{~cm}$ pericardial effusion with globally impaired left ventricular systolic contraction. He subsequently had a

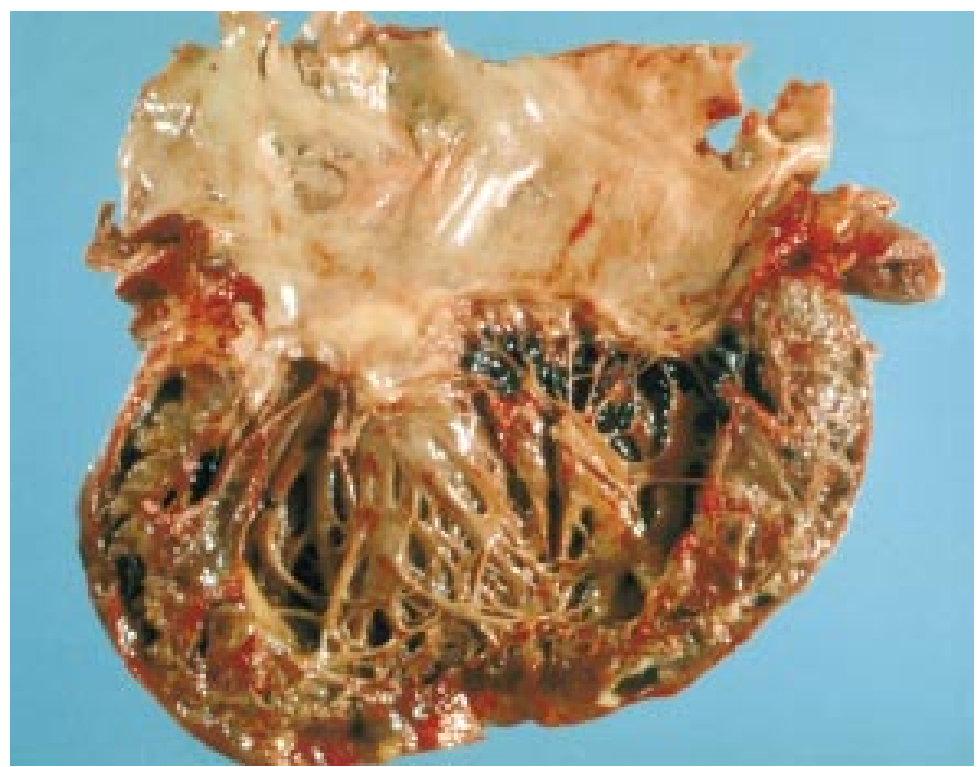

cardiac arrest due to electromechanical dissociation, and a pericardiocentesis of $20 \mathrm{ml}$ of blood stained fluid regained cardiac output. He died of circulatory failure that evening. All blood and pericardial fluid cultures were subsequently negative.

At necropsy there was a purulent pericardial effusion. The left ventricle was almost entirely replaced by multiple confluent abscesses, but without discernible perforation, and the right ventricle was normal. The heart valves were normal with no vegetations. There was moderate to severe occlusive coronary disease in all three arteries. A small right kidney had multiple small papillary abscesses. Bacteriological examination of the left ventricular and renal abscesses grew coliforms.

In cases of severe septicaemia myocardial abscesses are often clinically silent and only found at necropsy. Our patient had presented to his general practitioner with a urinary tract infection six months before hospital admission and remained clinically well in the intervening period. The finding of coliforms in both the left ventricular and renal abscesses suggests that subacute renal tract infection resulted in a bacteraemia with seeding of the myocardium leading to multiple unruptured myocardial abscesses.

LOUISA MCILWAINE STEPHEN STOTT DUNCAN HOGG 